\title{
Problemas de calidad en la evaluación de la investigación en Medicina Crítica en México
}

\author{
José Javier Elizalde González*
}

Varios son los problemas de la investigación en el campo de la Medicina Crítica en México y probablemente en otras latitudes. El contar con miembros expertos y participativos en el Comité Editorial y el efectuar una revisión minuciosa y exhaustiva por pares de los trabajos sometidos para publicación eventualmente no es suficiente.

En nuestro medio, padecimos por un largo tiempo de una cosecha escasa en cuanto a la cantidad de trabajos originales de investigación en el área, misma que ha madurado lo suficiente a lo largo de los años como para beneficiarse de un distinto escenario en el presente, con programas universitarios desde 1976, una Asociación Civil bajo la fortaleza y respaldo del Colegio Mexicano de Medicina Crítica (COMMEC), antes AMMCTI, que agremia la mayor parte de los especialistas en intensivos del país y que este año celebra en Puebla su XLV Congreso Anual; otra que congrega a las enfermeras especialistas en Medicina Crítica y en Terapia Intensiva y que cuenta en su haber con 42 Congresos Anuales, un sólido Consejo Mexicano de la especialidad desde 1986, que certifica la idoneidad de los egresados y es nuestro aval moral ante la sociedad mexicana, así como una gran cantidad de especialistas egresados de cerca de 40 sedes universitarias a lo largo del país, lo que le otorga a la Medicina Crítica de México una pujanza, vitalidad y dinamismo no antes vistos. Basta sencillamente con prestar atención a la gran cantidad de jóvenes especialistas que se aglomeran en las salas de conferencias de cursos y congresos de la especialidad para darse cuenta del distinto presente de la Terapia Intensiva de México.

Enfrentamos, sin embargo, nuevos retos, como lo manifestamos hace algún tiempo, al no desarrollarse en los distintos grupos de trabajo de nuestra nación verdaderas líneas de investigación y prevalecer cierto grado de desorden alrededor de la misma, aplicando el acelerador sólo ante la cercanía del Congreso Anual, aparte de exhibir un limitado interés en distintos aspectos éticos en torno a la investigación. En dicha ocasión, escribí que la investigación clínica no tiene como meta la presentación de trabajos en congresos médicos, sino la divulgación escrita del nuevo conocimiento científico comunicado a través de publicaciones especializadas que produzcan cambios y un avance en el conocimiento

\footnotetext{
* Editor de Medicina Crítica. INCMNSZ.

Este artículo puede ser consultado en versión completa en http://www.medigraphic.com/medicinacritica
}

en beneficio de la comunidad. ${ }^{1}$ Continuamos padeciendo de vez en vez de dobles sometimientos y, en ocasiones, de solicitudes de modificaciones de autoría, lo que deja suponer una nebulosa problemática aún mayor detrás de la gestión de la investigación y su ulterior divulgación en distintas instituciones. ${ }^{2}$

Otros problemas provienen del bajo nivel de originalidad de algunos proyectos de investigación, así como una mala calidad en la recolección de datos, pero sobre todo un diseño deficiente con un uso inapropiado de la metodología, destacando la falta de planeación que, para serlo verdaderamente, tiene que ser oportuna, con ausencia de un «plan de vuelo» en la forma de un protocolo escrito de investigación, analizado, consensuado y aprobado prospectivamente, primero, entre los propios investigadores y, luego, por las instancias reguladoras, según sea el caso, lo que redunda en múltiples problemas que lo limitan; entre otros factores, la práctica de reclutar pocos sujetos de investigación: estos tamaños muestrales muchas veces son insuficientes para la obtención de resultados robustos. ${ }^{3}$

Hemos observado que prevalece cierta confusión entre los diferentes idiomas de los números (nominal, ordinal, intervalar y de razón), lo que lleva a la incorrecta aplicación de distintas herramientas estadísticas, y también entre los distintos tipos de estudios de acuerdo al periodo en que se captan los datos y que sólo son dos: retrospectivo y prospectivo (el retrospectivo parcial en el que se cuenta con parte de la información, mientras que la restante está por obtenerse, se considera retrospectiva), entre lo que el estudio es de acuerdo con la evolución del mismo objeto de estudio, y que sólo puede ser longitudinal o transversal, así como entre lo que el estudio es acorde con la comparación de las poblaciones analizadas, que sólo puede ser descriptivo o comparativo y, finalmente, entre los distintos estudios de acuerdo con la intervención del investigador sobre el fenómeno estudiado, mismos que sólo pueden ser observacionales o experimentales. Éstos son los ejes fundamentales de los criterios de clasificación de la investigación clínica que podemos realizar, los cuales no son mutuamente excluyentes.

Como sabemos, el tipo de estudio de investigación debe elegirse sólo en función de los objetivos que se pretende alcanzar, de los propios recursos con los que se cuenta en el grupo de trabajo y del tipo específico de problema que se desea abordar.

Valga a manera de ejemplo lo acontecido con la investigación nacional de cuidados intensivos tomando 
como marco de referencia el actual XLV Congreso Anual y que llenará las páginas de la revista Medicina Crítica en los siguientes meses. Para este evento se aceptaron un total de 123 trabajos de investigación, de los cuales $42(34 \%)$ corresponden a presentación de casos que, sin demeritar de ninguna forma, no constituyen en sí una forma de investigación formal planeada y protocolizada, sino casos interesantes, complejos, a veces únicos, con los que el clínico puede tropezarse por azar y vale la pena difundir y discutir entre pares y eventualmente publicar. Para ello existe desde siempre una muy apreciada sección especial en nuestra revista, misma que se conservará. Algunos de estos casos se nos envían con un número mayor de autores que los mismos estudios multicéntricos (nueve versus siete autores, respectivamente, para este año). Las mayores áreas de interés son la respiratoria, con 23 trabajos de investigación (19\%), seguido por la neurológica con $10(8 \%)$ y la cardiovascular con nueve $(7.3 \%), 100 \%$ de esta última correspondiendo a casos clínicos. Veintiséis trabajos $(21 \%)$ se entregaron en extenso y fueron admitidos en el Concurso Académico «Mario Shapiro» 2018, de los cuales tres, los ganadores, tendrán una publicación expedita en Medicina Crítica. De éstos, que constituyen seguramente lo mejor de la producción del año, 11 (42\%) son retrospectivos, y los 15 restantes (58\%) prospectivos. Los primeros incluyeron en promedio 96 pacientes por estudio (rango 27 a 206 casos), en uno no se especifica el tamaño de la población. El tiempo promedio que se abarcó en la recolección de las variables analizadas fue de 47 meses (rango ocho a 102 meses). En cuanto a los prospectivos, incluyeron en promedio 108 pacientes por estudio (rango 12 a 171), en un periodo promedio muy corto, de apenas 8.5 meses (rango tres a 19 meses), en uno de ellos no se establece un periodo específico. Estos trabajos, además de problemas importantes de redacción, no tienen muchas veces el formato requerido para ser publicados, sino más bien el de una tesis de postgrado, con largas secciones de introducción que sobrepasan por mucho lo actualmente publicable; en otras ocasiones, carecen del resumen en inglés, palabras clave, sesión de derechos, etc., además de una forma incorrecta de acotar bibliografía tanto estándar como electrónica, todo lo que hace suponer un incompleto trabajo de supervisión y revisión en las respectivas sedes.

De los restantes 97 trabajos, sólo dos son multicéntricos (1.6\%); uno de ellos internacional, en el que no se establece el tamaño muestral en el resumen y consiste en la aplicación de un cuestionario, el otro realizado en cuatro hospitales, con una muestra de sólo 48 pacientes. De este grupo de trabajos de investigación, sólo 21 son prospectivos (17\%), en algunos no se establece en el resumen el tiempo que abarcaron (12/21, 57\%), el resto fue realizado en un tiempo promedio de cuatro meses y medio (rango tres a siete meses), por norma poco antes del Congreso Anual, con una población promedio de 144 pacientes por estudio (rango de cuatro a 200 casos); entre todos ellos, no se llega a los mil pacientes analizados de manera prospectiva. Extrapolando su capacidad de reclutamiento, algunos grupos podrían haber reunido prospectivamente más de mil casos en un periodo de 12 a 18 meses, o incluso más, y coordinarse y hacerlo de manera multicéntrica. Podemos hablar de varios miles de pacientes mexicanos estudiados de manera prospectiva en distintas áreas de la Medicina Crítica en dos o tres años, pero para eso se requiere trabajar en la mejora de ciertas áreas de oportunidad, como la planeación y el liderazgo.

En cuanto a los estudios retrospectivos de este año (28\%), en siete no se establece tamaño muestral; los restantes constan de una población estudiada promedio de 131 casos por estudio (rango de siete a más de mil sujetos estudiados), con un total de pacientes críticos incluidos en todos ellos de poco menos de 3,500; en una serie, se reportan 1,154 procedimientos en un número no precisado de pacientes a lo largo de 60 meses. En otros dos estudios, se reportan casos a lo largo de 60 y 90 meses, respectivamente; en el primero no se reporta el tamaño de la población y en el otro se reúnen sólo 42 casos, ambos son estudios con resultados negativos, en los que no se encuentra asociación entre las variables estudiadas. El tiempo promedio en el que este grupo de estudio recogió información fue de 41 meses, esto es 3.5 años (rango de tres meses a 7.5 años), muy superior al de los estudios prospectivos, lo que resulta lógico por la naturaleza de los estudios retrospectivos, donde, con fuerza de trabajo y los nuevos sistemas hospitalarios de tecnología de la información, se pueden realizar búsquedas extensas en un corto tiempo. Muchas de estas revisiones fueron iniciadas este año, generalmente en enero, y concluyeron hace poco (junio), lo que quizá indica, entre otras cosas, cierto grado de improvisación y apremio, en un medio saturado de fuertes responsabilidades asistenciales.

Lo anterior sin mencionar en muchos escritos el uso inapropiado del lenguaje por distintas razones, ${ }^{4} \mathrm{con}$ la invención de vocablos adaptados del inglés, como «ecoguiado» en lugar de «guiado por ultrasonido» o ventilado con «presión soporte» o con «volumen control» en lugar de «ventilado con soporte por presión» o «ventilado en modo controlado por volumen», etcétera.

Como podemos darnos cuenta, muchos son aún los inconvenientes y problemas de calidad, no sólo de cantidad, por resolver en la investigación de nuestra especialidad. Es obvio que necesitamos sentarnos a meditar y discutir sobre estos aspectos y, entre otras cosas, reforzar el conocimiento en metodología de la investigación en el ámbito de la Medicina Crítica del país y aprender a trabajar en equipo, sin menospreciar ni relegar los loables esfuerzos que ya se vienen haciendo 
por generar nuevo conocimiento en Medicina Intensiva en nuestro medio.

Una parte de la explicación recae posiblemente en el compromiso de los profesores encargados de los cursos de Medicina Crítica del país y a la inercia que toda una cultura institucional ejerce sobre los jóvenes médicos que ahí se forman y que no hacen otra cosa que reproducir lo que observan en sus superiores, por lo que los invitamos a discutir el contenido de esta editorial y la bibliografía que la acompaña en sus distintos grupos de trabajo y difundir el contenido del presente documento en un intento de corregir algunas de estas desviaciones en beneficio de todos, en especial de la Medicina Crítica de México.

\section{BIBLIOGRAFÍA}

1. Elizalde GJ. Sobre ética, líneas de investigación y otros problemas. Rev Asoc Mex Med Crit Ter Int. 2016;30(4):221.

2. Elizalde GJ. De autores y autoría. Rev Asoc Mex Med Crit Ter Int. 2016;30(3):150.

3. Elizalde GJ. Nuevos retos. Rev Asoc Mex Med Crit Ter Int. 2016;30(1):6-7.

4. Elizalde GJ. Presión de impulsión. Med Crit. 2018;32(1):6. 